\title{
Contactless Distribution Path Optimization Based on Improved Ant Colony Algorithm
}

\author{
Feng $W u$ i \\ College of Information Engineering, Xinyang Agriculture and Forestry University, Xinyang 464000, China \\ Correspondence should be addressed to Feng Wu; 3306792320@qq.com
}

Received 22 February 2021; Accepted 16 May 2021; Published 24 May 2021

Academic Editor: Yandong $\mathrm{He}$

Copyright (C) 2021 Feng Wu. This is an open access article distributed under the Creative Commons Attribution License, which permits unrestricted use, distribution, and reproduction in any medium, provided the original work is properly cited.

\begin{abstract}
In the context of the normalization of the epidemic, contactless delivery is becoming one of the most concerned research areas. In the severe epidemic environment, due to the frequent encounter of bayonet temperature measurement, road closure, and other factors, the real-time change frequency of each traffic information is high. In order to improve the efficiency of contactless distribution and enhance user satisfaction, this paper proposes a contactless distribution path optimization algorithm based on improved ant colony algorithm. First of all, the possible traffic factors in the epidemic environment were analyzed, and the cost of each link in the distribution process was modeled. Then, the customer satisfaction is analyzed according to the customer service time window and transformed into a cost model. Finally, the total delivery cost and user satisfaction cost were taken as the optimization objectives, and a new pheromone updating method was adopted and the traditional ant colony algorithm was improved. In the experiment, the effectiveness of the proposed model and algorithm is verified through the simulation optimization and comparative analysis of an example.
\end{abstract}

\section{Introduction}

Contactless distribution refers to placing goods in a designated location, such as delivery cabinets and doorsteps. Customers then pick up the goods themselves, thus achieving contactless delivery of goods. Compared with traditional delivery methods, contactless delivery reduces the contact between people [1]. During the COVID-19 outbreak, contactless delivery effectively reduced the risk of cross-infection between delivery personnel and customers. In the context of the normalization of the epidemic, the demand for contactless delivery is increasing day by day. When the epidemic control policy is strict, considering the factors of safety and necessity of life, most of the goods distributed without contact are fresh goods. Therefore, the contactless distribution of fresh goods has important research significance. Reasonable planning of fresh goods logistics distribution path not only can reduce the logistics distribution cost and ensure the freshness of fresh products, but also can increase customer satisfaction and enhance the competitiveness of enterprises.
Since the vehicle routing problem was proposed [2], a large number of research achievements have been generated. Desrocherst and Verhoog [3] proposed a hybrid vehicle path model. Solomon and Desrosiers incorporated the concept of time windows into the vehicle routing problem [4]. On this basis, Jabali et al. solved the time window constraint [5] by punishing the cost, introduced the concepts of soft time window and hard time window and established the vehicle routing problem of soft time window. In recent years, advocating green development, with the goal of reducing energy consumption and carbon emissions, green vehicle routing has attracted the attention of academic circles. Tabu search algorithms based on particle swarm optimization (PSO), simulated annealing, and path partition are often used to solve the green vehicle routing problem model [6]. In order to highlight the impact of vehicle energy consumption and carbon emissions on the environment, literature [7] puts forward the problem of pollution path. Literature [8] analyzed the influence of vehicle departure time on vehicle speed, built a time-dependent single target pollution path model, and solved it by using tabu search algorithm and 
departure time and speed optimization algorithm, respectively. Literature [9] designed a Wolf swarm algorithm to solve the vehicle routing problem of multiple distribution centers. Yan et al. [10] studied the two-dimensional packing vehicle routing problem including time window and multiple vehicle field factors and proposed the quantum particle swarm optimization algorithm to solve the problem. Literature [11] designed a variable neighborhood search algorithm for solving the VRP model. Literature [12] used dynamic programming algorithm to solve the vehicle routing problem.

The research on the contactless distribution path of fresh goods is mainly applied to the field of cold chain logistics and transportation. At present, there have been a series of research results in the field of cold chain logistics and transportation. Tarantilis and Kiranoudis [13] took the distribution of meat and milk in Greece as an example to study the vehicle routing problem of open cold chain logistics distribution with multiple models and multiple distribution centers. Amorim et al. [14] constructed a multitime window and multivehicle routing problem for a food distribution problem in Portugal. Yang and Sun [15] studied the VRP model of perishable goods in cold chain logistics and optimized the distribution path by using genetic algorithm. Zhang et al. [16] designed a mathematical model of vehicle path of cold chain logistics with multiple depots and models and solved it through genetic algorithm and elite selection method. Ma et al. [17] studied the vehicle routing optimization model of cold chain logistics based on random demand. Lan et al. [18] analyzed the impact of urban road congestion on the vehicle distribution cost of cold chain logistics, built the corresponding vehicle path optimization model, and designed a hybrid genetic algorithm to solve the problem. In the case of uncertain demand, Li and Wang [19] established the distribution path optimization problem of perishable products with time windows with random customer demand.

To sum up, research on the distribution of vehicles in cold chain logistics has produced certain research results, which lays a good foundation for the research on the green vehicle routing problem in cold chain logistics. However, there are also some limitations, mainly reflected in the following three aspects: (1) The existing cold chain logistics distribution does not consider the real-time impact of road closure, bayonet temperature measurement, and traffic congestion on path planning. (2) At present, the relevant literature of cold chain logistics vehicle routing optimization mainly discusses the economy, and seldom involves the green benefits of vehicle energy saving and emission reduction. (3) Less consideration is given to the impact on user satisfaction.

On this basis, the possible traffic factors in the epidemic environment are analyzed in this paper, and the cost of each link is modeled in the distribution process. The fixed cost, transportation cost, refrigeration cost, carbon emission cost, penalty cost, and user satisfaction cost of the vehicle were taken as objective functions to build the model. Based on the traditional ant colony algorithm, the updating method of pheromone is improved, and a path planning algorithm which is more consistent with the contactless distribution model is obtained. The improved post-ant colony algorithm for path planning can adjust the path according to the realtime traffic situation, which can not only improve the efficiency of contactless distribution, but also improve user satisfaction.

\section{Distribution Problem Modeling}

2.1. Description of Distribution Problem. In actual application scenarios, distribution companies will not set up too many distribution centers due to cost constraints [20]. In an epidemic situation, the number of distribution centers set up will be more limited. Therefore, in order to be more consistent with the actual situation, the hypothetical situation of the distribution problem in this paper is as follows.

(1) There is only one distribution center, which has multiple distribution vehicles.

(2) The distribution scope is the residential areas, shopping malls, and other places near the distribution center. The same community or shopping mall will have multiple goods at the same time distribution needs. For the convenience of description, the same residential area, shopping mall, and other residential areas are collectively referred to as the same residential area in the following description.

(3) The location coordinates of each customer can be accurate to the specific community, the demand of each customer is known, and the sum of the demand of the same community is known.

(4) In order to save distribution resources, generally only one vehicle will be arranged to meet the needs of customers in the same location at a time; that is, customers in the same community will only be provided with distribution services by one delivery vehicle.

(5) In this paper, it is assumed that each vehicle starts from the distribution center and returns to the distribution center after completing certain distribution tasks. During the distribution process, the vehicle is only responsible for delivering goods and contacting customers for self-collection. Delivery personnel are not responsible for face-to-face inspection, return, and other services.

(6) If the goods are not delivered within the specified time, its quality will decline, and the customer can ask for compensation after receiving the goods. Therefore, if the delivery is not within the time frame required by the customer, there will be a penalty fee.

(7) Assume that the distribution center has enough vehicles to complete all the distribution tasks, and the distribution vehicles have the same model and are equipped with refrigeration equipment. The maximum loading capacity of the vehicle is known. The customer demand on each distribution route 
cannot exceed the maximum loading capacity of the distribution vehicle.

Based on the above assumptions, the cold chain distribution problem of contactless distribution studied in this paper can be described as follows. A fresh goods distribution center distributes fresh goods to the surrounding customer groups, using refrigerated trucks as transportation tools. The demand and geographical location of each customer is known, and each customer has a certain limit on the delivery time of goods. The vehicle shall return to the distribution center after completing certain distribution tasks. In order to optimize the objective function, the driving route is arranged reasonably under the condition of satisfying the customer's demand. Figure 1 is a basic distribution diagram of the problem.

\subsection{Signs and Variables}

$M$ : the number of communities served by the logistics distribution center

$M_{i}$ : the number of customers in the $i$ th community

$N$ : the number of customers served by the logistics distribution center

$W$ : the number of refrigerated vans owned by the distribution center

$d_{i j}$ : the distance that vehicles travel directly from community $i$ to community $j$

$q_{i}$ : the quantity demanded by all customers in community $i$

$p$ : unit price of fresh goods

$Q_{w}$ : maximum load capacity of the delivery vehicle

$Q_{i j}$ : the load of a vehicle when it travels directly from community $i$ to community $j$

$\rho\left(Q_{i j}\right)$ : fuel consumption per unit distance of goods transporting $Q_{i j}$ when the vehicle travels from community $i$ to community $j$

$t_{i}^{k}$ : the time it takes for vehicle $k$ to reach customer $i$ $U\left(t_{i}\right)$ : customer's $i$ satisfaction with goods distribution $\left[\mathrm{ET}_{i}, \mathrm{LT}_{i}\right]$ : the best service window for customer $i$ $\left[\mathrm{ET}_{i}^{*}, \mathrm{LT}_{i}^{*}\right]$ : the service time window acceptable to customer $i$

$\varepsilon_{1}$ : penalty cost per unit time of the vehicle serving customer $i$ before the time $\mathrm{ET}_{i}$

$\varepsilon_{2}$ : penalty cost per unit time of the vehicle serving customer $i$ after the time $\mathrm{LT}_{i}$

$t_{i}$ : the time that vehicle $k$ serves customer $i$, where $t_{0}=0$ $\alpha_{i j k}$ : a path factor; when vehicle $k$ drives directly from customer $i$ to customer $j$, its value is 1 ; otherwise, its value is 0

$\beta_{i k}$ : the vehicle distribution factor; when the goods of customer $i$ are delivered by vehicle $k$, the value is 1 ; otherwise, the value is 0

$C 1$ : fixed operating cost of the vehicle
C2: unit transport cost of the vehicle

C3: environmental cost per unit $\mathrm{CO}_{2}$ of emissions consumed

$C_{e 1}$ : refrigeration cost per unit time during the distribution process

$C_{e 2}$ : refrigeration cost per unit time during loading and unloading

$\omega$ : the emission coefficient of $\mathrm{CO}_{2}$

$r_{i j}$ : the road condition factor of vehicles driving directly from community $i$ to community $j$

2.3. Road Traffic Factor. In the severe epidemic environment, due to the frequent encounter of bayonet temperature measurement, road closure, and other factors, the real-time performance of each road condition information changes greatly. In order to improve the efficiency of contactless distribution, these factors need to be considered in the distribution path optimization model. In order to meet the practical application scenarios and facilitate the establishment of the subsequent cost model, the road traffic situation is divided into four situations.

Situation 1: the road condition is good and does not affect the normal work of the delivery vehicle. In this case, the road condition factor $r_{i j}$ is constant 1 .

Situation 2: the road condition is good, but there is a temperature measuring bayonet in the road section of the distribution vehicle. Because the temperature measurement and registration of bayonet need a certain time, the distribution of vehicles will be affected to a certain extent. At this point, the road condition factor $r_{i j}$ is a constant greater than 1 , and its size is related to the number of specific temperature measuring bayonets. Here, the temperature measurement bayonet information can be obtained by the relevant government departments. In general, temperature bayonet conditions do not change very frequently and generally do not change on the same day, such as $r_{i j} \in(1,3]$.

Situation 3: the road condition is normal, and the road section of the delivery vehicle will encounter congestion. In this case, the road condition factor $r_{i j}$ is a variable greater than 1 , and the specific value is determined by the degree of traffic congestion. Traffic congestion is assumed to be captured by real-time information released by the Department of Transportation. In general, traffic jams are highly correlated with the time of day. For example, in the morning and evening peak hours, when the road condition is poor, the value of the road condition factor is large, such as $r_{i j} \in(3,5]$.

Situation 4: in case of severe epidemic, some road sections may be closed in order to control access to the area. When the road is closed, in order to realize the distribution of goods, only a detour can be taken. In this case, the road condition factor $r_{i j}$ will be reassigned according to the detour distance and the traffic 


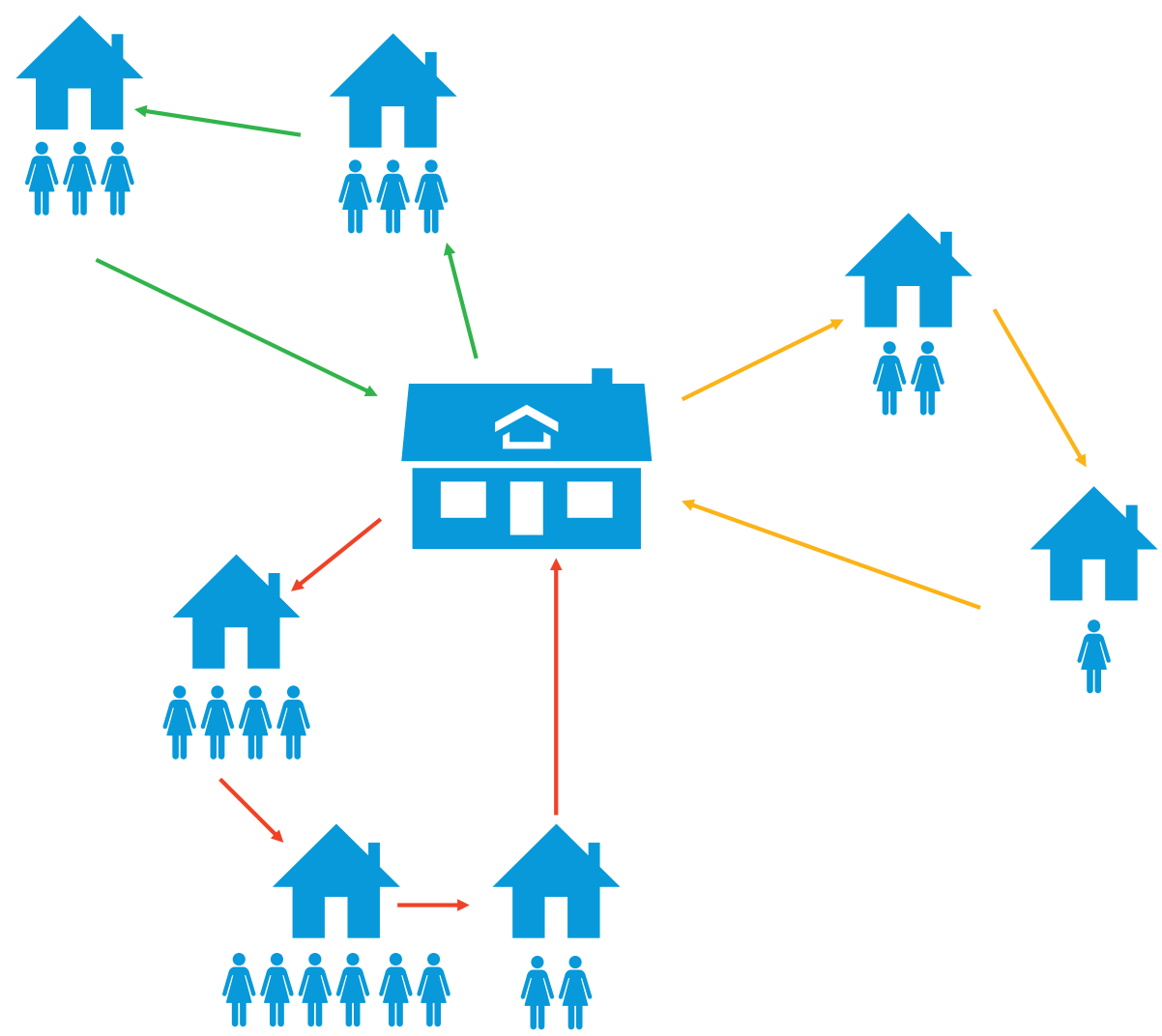

Figure 1: The basic distribution diagram.

condition, and its value is usually larger, such as $r_{i j} \in(5,10]$.

2.4. Distribution Cost Analysis. In the context of epidemic environment, in order to better reflect the actual situation of contactless distribution, this paper adds carbon emission, customer satisfaction, and traffic conditions on the basis of vehicle cost, refrigeration cost, and penalty cost. Among them, carbon emission and customer satisfaction are calculated as separate cost functions. Route traffic factors affect the cost of each of the above items, but in order to simplify the calculation model, this paper converts the path traffic factors into the influence coefficient of distance. That is, when the road conditions encounter temperature checkpoints, traffic jams, and road closures, the distance assumption becomes larger, so that the vehicle cost, refrigeration cost, and time cost will all increase.

2.4.1. Fixed Costs of Vehicles. The fixed cost of using a vehicle is usually constant, including fixed wear and tear on the vehicle and costs related to the use of the vehicle, such as the driver's salary, independent of the vehicle's mileage and the number of customers served. The fixed cost $V 1$ of the vehicle is shown as follows:

$$
V 1=C 1 \sum_{j=1}^{M} \sum_{k=1}^{W} \alpha_{0 j k} .
$$

2.4.2. Transportation Costs of Vehicles. The transportation cost of a vehicle mainly refers to the fuel consumption cost, which is usually proportional to the mileage of a vehicle. Therefore, the transportation cost $V 2$ of a vehicle can be expressed as follows:

$$
V 2=C 2 \sum_{k=1}^{W} \sum_{i=0}^{M} \sum_{j=0}^{M} r_{i j} d_{i j} \alpha_{i j k} .
$$

2.4.3. Costs of Refrigeration. The refrigeration cost should be considered in the transportation process of cold chain model. Cooling costs include the energy consumed to keep the vehicle cool during transportation and the cost of additional energy provided by the cooling system during offloading.

Therefore, in the process of transportation, the refrigeration cost $V 31$ of the refrigerated truck is shown as follows:

$$
V 31=C_{e 1} \sum_{k=1}^{W} \sum_{i=0}^{M} \sum_{j=0}^{M} \alpha_{i j k} t_{i j}^{k} .
$$

During the unloading process, the refrigeration cost V32 of the refrigerated truck can be expressed as follows:

$$
V 32=C_{e 2} \sum_{k=1}^{W} \sum_{i=0}^{M} \beta_{j k} \omega_{j} .
$$

Therefore, the refrigeration cost of the whole process is 


$$
V 3=V 31+V 32=C_{e 1} \sum_{k=1}^{W} \sum_{i=0}^{M} \sum_{j=0}^{M} \alpha_{i j k} t_{i j}^{k}+C_{e 2} \sum_{k=1}^{W} \sum_{i=0}^{M} \beta_{j k} \omega_{j}
$$

2.4.4. Costs of Carbon Emission. Carbon emission cost mainly refers to the $\mathrm{CO}_{2}$ emission cost generated by vehicle fuel consumption in the distribution process, carbon emission $=$ fuel consumption $\times \mathrm{CO}_{2}$ emission coefficient . Fuel consumption is related to both transport distance and vehicle load capacity. The fuel consumption per unit distance $\rho$ can be expressed as a linear function dependent on the cargo carrying capacity of the truck $X$. Divide the total vehicle weight into vehicle dead weight $Q_{0}$ and cargo capacity $X$; then the unit distance fuel consumption $\rho(X)$ is shown as follows:

$$
\rho(X)=a\left(Q_{0}+X\right)+b .
$$

The maximum cargo capacity of the vehicle is set as $Q$, the fuel consumption per unit distance is $\rho^{*}$ when full load, and the fuel consumption per unit distance is $\rho_{0}$ when no load.

$$
\begin{aligned}
\rho_{0} & =a Q_{0}+b, \\
\rho^{*} & =a\left(Q_{0}+Q\right)+b, \\
a & =\frac{\rho^{*}-\rho_{0}}{Q} .
\end{aligned}
$$

Thus, fuel consumption per unit distance $\rho(X)$ can be expressed as

$$
\rho(X)=\rho_{0}+\frac{\rho^{*}-\rho_{0}}{Q} X .
$$

Therefore, in the process of fresh agricultural products distribution, if the goods of $i j$ are transported from customer $i$ to customer $j$, then the carbon emissions generated when driving between $(i, j)$ can be expressed as

$$
E_{1}=\omega \rho\left(Q_{i j}\right) r_{i j} d_{i j}
$$

where $\omega$ is the $\mathrm{CO}_{2}$ emission coefficient, $Q_{i j}$ represents the deadweight when the vehicle travels directly from customer $i$ to customer $j, \rho\left(Q_{i j}\right)$ represents the fuel consumption per unit distance when the vehicle travels between $(i, j)$, and the deadweight is $Q_{i j}$. In this paper, the carbon emission cost is calculated through the carbon tax mechanism, that is, carbon emission cost $=$ carbon $\operatorname{tax} \times$ carbon emission. If the carbon tax is set as $C 3$, then the total carbon emission cost $V 4$ in the distribution process can be expressed as

$$
V 4=C 3 \cdot \omega \sum_{k=1}^{W} \sum_{i=0}^{M} \sum_{j=0}^{M} r_{i j} d_{i j} \alpha_{i j k} \rho\left(Q_{i j}\right) .
$$

2.4.5. Penalty Costs. The quality of fresh products is greatly affected by the time factor, so in the process of distribution, each customer has characteristic requirements for the service time window, which is divided into acceptable service time window $\left[\mathrm{ET}_{i}^{*}, \mathrm{LT}_{i}^{*}\right]$ and optimal service time window $\left[\mathrm{ET}_{i}, \mathrm{LT}_{i}\right]$ according to the service level. If the delivery time is not within the optimal delivery time, it may lead to the loss of subsequent business. At the same time, the optimal time window is related to the time when distribution starts to serve, so the penalty cost function can be expressed as

$$
V 5\left(t_{i}^{k}\right)= \begin{cases}\operatorname{Max} E, & t_{i}^{k}<\mathrm{ET}_{i}^{*}, \\ \varepsilon_{1}\left(\mathrm{ET}_{i}-t_{i}^{k}\right), & \mathrm{ET}_{i}^{*} \leq t_{i}^{k}<\mathrm{ET}_{i}, \\ 0, & \mathrm{ET}_{i} \leq t_{i}^{k} \leq \mathrm{LT}_{i}, \\ \varepsilon_{2}\left(t_{i}^{k}-\mathrm{LT}_{i}\right), & \mathrm{LT}_{i}<t_{i}^{k} \leq \mathrm{LT}_{i}^{*}, \\ \operatorname{Max} E, & t_{i}^{k}>\mathrm{LT}_{i}^{*} .\end{cases}
$$

where $\operatorname{Max} E$ is a constant, representing the maximum loss caused outside the acceptable delivery time window.

2.4.6. User Satisfaction. The customer satisfaction with the service $U\left(t_{i}^{k}\right)$ also depends on the time window when the customer starts the service. The specific function is as follows:

$$
U\left(t_{i}^{k}\right)= \begin{cases}0, & t_{i}^{k}<\mathrm{ET}_{i}^{*}, \\ \frac{t_{i}^{k}-\mathrm{ET}_{i}^{*}}{\mathrm{ET}_{i}-\mathrm{ET}_{i}^{*},} & \mathrm{ET}_{i}^{*} \leq t_{i}^{k}<\mathrm{ET}_{i}, \\ 1, & \mathrm{ET}_{i} \leq t_{i}^{k} \leq \mathrm{LT}_{i}, \\ \frac{\mathrm{LT}_{i}^{*}-t_{i}^{k}}{\mathrm{LT}_{i}^{*}-\mathrm{LT}_{i},} & \mathrm{LT}_{i}<t_{i}^{k} \leq \mathrm{LT}_{i}^{*}, \\ 0, & t_{i}^{k}>\mathrm{LT}_{i}^{*} .\end{cases}
$$

In order to take user satisfaction into account in the overall loss function, the loss cost of user satisfaction is defined as follows:

$$
V 6=\sum_{k=1}^{W} \sum_{i=1}^{N} \frac{1}{1+U\left(t_{i}^{k}\right)}
$$

2.5. Construction of Distribution Model. To sum up, considering the influence of traffic path factors and user satisfaction, the final optimization model of distribution path is as follows:

$$
\min V=V 1+V 2+V 3+V 4+V 5+V 6
$$

where 


$$
\begin{aligned}
& \sum_{i=1}^{M} q_{i} \beta_{i k} \leq Q_{k}, \\
& \sum_{k=1}^{W} \beta_{i k}=1, \\
& \sum_{i=0}^{M} \alpha_{i j k}=\beta_{j k}, \\
& \sum_{j=0}^{M} \alpha_{i j k}=\beta_{i k}, \\
& \sum_{i, j \in S \times S} \alpha_{i j k} \leq|S|-1, \quad S \in\{1,2, \ldots, N\}, \\
& t_{j}=t_{i}+t_{i j},
\end{aligned}
$$

where the objective equation (14) represents the comprehensive cost formula of cold chain logistics transportation including carbon emissions. Equation (15) is the constraint of vehicle carrying capacity. Equation (16) ensures that each customer is only served by one vehicle. Equations (17) and (18) restrict that for any customer, only one vehicle can arrive and depart once. Equation (19) is the elimination condition of secondary loop. Equation (20) represents the continuity of the distribution process.

\section{Improved Ant Colony Algorithm}

Because VRP belongs to NP-hard problem, heuristic algorithm is usually used to solve it. The optimization of the distribution path of fresh agricultural products considering carbon emission is also a NP-hard problem. Because its complexity is higher than that of VRP, the algorithm is required to solve it. Ant colony algorithm is a kind of simulated evolutionary algorithm inspired by real ant colony behavior in nature. Ant colony algorithm has shown good performance in many aspects, such as positive feedback, strong robustness, distributed computing, and easy to be combined with other methods. Its positive information feedback mechanism enables it to quickly find better solutions. Distributed computing makes it easy to implement algorithms in parallel and avoid falling into local optimality. Therefore, this paper uses the improved ant colony algorithm to solve the model.

3.1. Basic Step of the Algorithm. The basic flow of ant colony algorithm is as follows.

Step 1. Initialize parameters. There are $m$ ants; start from the distribution center at the same time and select the first access point according to certain conditions, respectively.

Step 2. List all unvisited points for each ant and set the number of them as $S 1$.
Step 3. Judge the value of $S 1$. If $S 1=0$, it means that the ant has completed all the distribution tasks and should return to the distribution center to end the distribution of this line. If $S 1>0$, then $S 2(S 2 \leq S 1)$ is selected from $S 1$ unaccessed points that meet the access conditions. If $S 2=0$, it means that no optional point meets the conditions, and the vehicle returns to the distribution center. If $S 2>0$, then $S=\min (S 1, S 2)$, and add the distribution center to the candidate access points. Select one point as the next service point according to a certain probability, and return to Step 2.

Step 4. Judge whether all ants have completed the delivery task. If so, calculate the total route length of each ant, select the shortest route and record it, and update the pheromone according to the rules.

Step 5. Calculate the minimum value of routes passed by each ant in each operation, denoted as $L_{i}(i=1,2, \ldots$, $N)$; after multiple calculations, the iterative optimal solution can be obtained as $\min \left(L_{i}\right)$.

3.2. Heuristic Factor. Heuristic factor is the key factor inspiring ant to select nodes and the core part of ant colony algorithm, which can directly affect the algorithm's solution quality. The influence of vehicle load is taken into account in the model built for the optimization of fresh agricultural products distribution path considering carbon emissions. If only the distance is taken as a heuristic factor, the solution results will be affected, resulting in fuel waste and cost increase. Therefore, the customer's demand for goods is introduced into the heuristic factor, which makes the algorithm take into account the influencing factors of fuel consumption when selecting the next node. The heuristic factors for the optimization of the distribution path of fresh agricultural products considering carbon emissions are shown as follows:

$$
\varphi_{i j}=\frac{q_{j}}{d_{i j}} .
$$

As shown in equation (21), on the premise of satisfying the vehicle load and time constraints, the ant individual will comprehensively calculate the heuristic factor according to the current load and distance. The distance is in the denominator, so the smaller the distance, the larger the heuristic factor, and the greater the probability that the ant will choose this customer point. Similarly, the load weight is at the molecular position, so the greater the demand for goods at the next customer point is, the greater the value of heuristic factor is, and the greater the selection probability is. In other words, the customer point with large demand is given priority for delivery, so as to reduce the load weight of the delivery vehicle and reduce fuel consumption.

3.3. Improved Pheromone Update Strategy. In the classical model, the specific updating mode of the pheromone left by the $k$ th ant from $i$ to $j$ is as follows [21].

The ant quantity system is 


$$
\Delta \tau_{k i j}=\left\{\begin{array}{l}
\frac{Q}{d_{i j}} \\
0 .
\end{array}\right.
$$

The ant density system is

$$
\Delta \tau_{k i j}=\left\{\begin{array}{l}
Q, \\
0 .
\end{array}\right.
$$

The ant circle system is

$$
\Delta \tau_{k i j}=\left\{\begin{array}{l}
\frac{Q}{L^{k}} \\
0 .
\end{array}\right.
$$

Inspired by the ideas of genetic algorithm, such as genetic inheritance, recombination and mutation, a new pheromone updating method is proposed in this paper. The new pheromone updating method can effectively retain the better route segments and combine reasonably between different route segments, so as to facilitate the rapid generation of the optimal solution. The pheromone left by the $k$ th ant from $i$ to $j$ changes to

$$
\Delta \tau_{k i j}=\left\{\begin{array}{l}
\left(K_{k i j}\right)^{\omega 1}\left(L_{k i j}\right)^{\omega 2}\left(R_{k i j}\right)^{\omega 3}\left(d_{k i j}\right)^{\omega 4}+\frac{Q}{R_{k}}, \\
0,
\end{array}\right.
$$

where $\omega 1, \omega 2, \omega 3$, and $\omega 4$ need to be determined according to specific problems. In general, $\omega 1$ is positive, $\omega 2, \omega 3$, and $\omega 4$ are negative, and $\omega 1=2.0, \omega 2=\omega 3=\omega 4=-0.5$ are used in the calculation of this paper. The value of equations (22)-(25) is based on whether the $k$ ant passes through sections $i$ and $j$. follows:

After improvement, new symbols need to be defined as

$K_{k i j}$ : the number of temperature measuring points at bayonet of the subline where the segment from point $i$ to point $j$ passed by the $k$ ant

$L_{k i j}$ : the length of the subline from point $i$ to point $j$ that the $k$ ant passes

$R_{k}$ : the total length of the route traveled by the $k$ ant

The indicator $K_{k i j}$ can strengthen the pheromone in the excellent subsegments. The indicator $L_{k i j}$ can distinguish between subpaths with the same or similar number of access points. Indicator $R_{k}$ reflects the advantages and disadvantages of the general routes passed by different ants. Through such pheromone updating method, the unblocked road sections can fully retain the relative stability, thus generating a better combination. On this basis, a small correction factor is added to all the road pheromones to ensure certain variation effect and avoid the algorithm falling into local optimal prematurely.

$$
p_{k}(i, j)= \begin{cases}\frac{\left[\tau(i, j)^{\alpha}\right]\left[\eta(i, j)^{\beta}\right]}{\sum_{\mu \in J_{k}(i)}\left[\tau(i, j)^{\alpha}\right]\left[\eta(i, j)^{\beta}\right]}, & j \in J_{k}(i), \\ 0, & j \notin J_{k}(i),\end{cases}
$$

$$
\begin{gathered}
\tau(i, j)=(1-\rho) \times \tau(i, j)+\sum_{k=1}^{m} \Delta \tau_{k}(i, j), \\
\tau(i, j)=\tau(i, j)+\tau_{0} .
\end{gathered}
$$

where $\alpha$ is the relative importance of residual information and $\beta$ is the relative importance of heuristic information. In this paper, let $\alpha=1, \beta=1$. $\rho$ is the pheromone volatilization parameter; $\rho$ is set as $0.5 . \tau_{0}$ is the pheromone correction factor to increase the probability of choosing different routes and avoid the precocity of the algorithm. In this paper, $\tau_{0}$ is set as 0.1. Equations (25)-(28) represent the updating steps of pheromone. Equation (26) represents the probability that the $k$ th delivery vehicle starts from community $i$ and takes different community $j$ as the target point.

The 2-opt local optimization algorithm is mixed into the ant colony algorithm. After all ants have completed the construction of the solution, the best solution of each generation is locally improved before the pheromone on each path is updated, so as to accelerate the convergence speed of the ant colony algorithm.

\section{The Experiment}

4.1. The Experimental Setup. In consideration of various factors of vehicle distribution in cold chain logistics, data of class $C$ (centralized distribution), class $R$ (random distribution), and class RC (mixed distribution) in Solomon's VRPTW database were used as the test data [22] in this paper. Each example had 100 customer points and 1 distribution center. In order to better fit the reality, the initial time of the model is set as 6:00 a.m., which is the earliest service time of the distribution center and is set as time 0 . According to the law of urban traffic, 7:00 to 9:00 and 17:00 to $19: 00$ in the afternoon are set as traffic jam periods, and the traffic in other periods is smooth. The temperature measuring bayonet points are randomly distributed on each route, and there is only one at most for each plot location, and the road is closed at most once for each plot location. The selected vehicle no-load weight is $5000 \mathrm{~kg}$; capacity is 1000 weight units. If a weight unit is $2 \mathrm{~kg}$, then the vehicle capacity is $2 \mathrm{t}$, and the corresponding carbon emission rate coefficient is $\omega 0=110, \omega 1=0, \omega 2=0, \omega 3=3.75 \times 10^{-4}$, $\omega 4=8702, \omega 5=0$, and $\omega 6=0$. Corresponding coefficient of load correction factor for [22] is $\chi_{0}=1.27$, $\chi_{1}=0.0614, \chi_{2}=0, \chi_{3}=0.001 \quad 1, \chi_{4}=-0.00235, \chi_{5}=0$, $\chi_{6}=0$, and $\chi_{7}=-1.33$. The values of other parameters involved in the model are shown in Table 1 , and those of algorithm parameters are shown in Table 2 [8]. The algorithm was programmed by MATLAB R2020 and calculated on 16 GB PC with CPU3.0 GHz memory. 
TABLE 1: Model parameter values.

\begin{tabular}{lc}
\hline Parameter & Parameter value \\
\hline$g_{k}$ & 150 yuan $/ \mathrm{units}$ \\
$\mu$ & $5 \mathrm{yuan} / \mathrm{h}$ \\
$\psi$ & $10 \mathrm{yuan} / \mathrm{h}$ \\
$p$ & $5 \mathrm{yuan} / \mathrm{kg}$ \\
$g f$ & $7 \mathrm{yuan} / \mathrm{L}$ \\
$c f$ & $0.0528 \mathrm{yuan} / \mathrm{kg}$ \\
$\theta_{1}$ & $0.0010 \mathrm{~h}$ \\
$\theta_{2}$ & $0.0015 \mathrm{~h}$ \\
$e z$ & $0.0066 \mathrm{~g} / \mathrm{kg} \cdot \mathrm{km})$ \\
$\theta_{3}$ & $15 \mathrm{yuan} / \mathrm{h}$ \\
$\theta_{4}$ & $20 \mathrm{yuan} / \mathrm{h}$ \\
$c h$ & $12 \mathrm{yuan} / \mathrm{h}$ \\
\hline
\end{tabular}

TABLE 2: Ant colony algorithm parameters.

\begin{tabular}{lc}
\hline Parameter & Parameter value \\
\hline NCmin & 40 \\
NCmax & 400 \\
$M$ & 15 \\
$\alpha$ & 1 \\
$\beta$ & 3 \\
$\gamma$ & 3 \\
$\varphi$ & 2 \\
$Q$ & 100 \\
\hline
\end{tabular}

4.2. Large Data Case for Path Optimization. The RC204 dataset was used to calculate path optimization. Its program running time is $290.3 \mathrm{~s}$, and the total distribution cost is 10052.8 yuan. A total of 9 vehicles are used for distribution, and the cost of manpower and vehicle use is 2943.9 yuan, the cost of refrigeration is 2656.6 yuan, the cost of fuel consumption is 3904.1 yuan, the cost of carbon emission is 50.1 yuan, the cost of waiting for punishment at the customer point early is 395.7 yuan, and the cost of customer satisfaction loss is 102.4 yuan. The specific path optimization scheme is shown in Table 3. In Table 3, SN represents the vehicle number, VR (vehicle driving) represents the vehicle driving path (0 represents the distribution center and 1-100 represents the customer point), and VAT (vehicle driving time) represents the time of the vehicle arriving at each customer point.

The calculation results show the following contents:

(1) The algorithm in this paper can obtain the optimal planning path in a relatively short time.

(2) By combining VR and VAT, it can be seen that due to the influence of road factors, customer service time window, vehicle load, and other constraints, there are obvious differences in the distribution routes of cold chain logistics vehicles. Vehicle 2 served the largest number of customer points, reaching 21. Vehicles 8 and 9 serve the least number of customer points. The reason for this is that each requirement point has a different time window, and vehicle routing planning must meet customer point time window requirements while ensuring minimum fitness.
(3) From VAT, it can be seen that both vehicle 1 and vehicle 2 start to distribute from the distribution center at 0 , while the departure time of other vehicles is different. If you start at 0 , you need to bear a large time window penalty. It shows that it is necessary to consider time dependence when planning the path of logistics enterprises. The route should be planned scientifically according to the actual situation of the road network, the time window of the customer points, and the cost of refrigeration and fuel consumption in the transportation process.

(4) According to VAT, vehicle 4 completely avoids the congestion period. Vehicles $1,3,6,7$, and 8 only enter the morning rush hour, not the evening rush hour. However, vehicles 2, 5, and 9 only entered the evening rush hour and did not enter the morning rush hour. This indicates that the method proposed in this paper can reasonably avoid the traffic jam period and improve the efficiency of vehicle distribution.

4.3. Path Planning for Different Distribution Examples. Different types of dataset were used to calculate the path optimization. Each dataset was calculated 10 times, and the average value was taken as the final result. The experimental results are shown in Table 4, where EX represents the type of calculation example, TC represents the total cost of distribution, TMC represents the cost of manpower and vehicle start-up and rental, CC represents refrigeration cost, FC represents fuel consumption, $\mathrm{CF}$ represents carbon emission cost, PC represents the penalty cost of time window, UC represents the cost of customer satisfaction loss, and VN represents the number of vehicles.

As shown in Figure 2 and Table 4, the following can be obtained:

(1) In the solution process of different calculation examples, the algorithm can reach the optimal solution and achieve stability within 400 iterations. It indicates that the algorithm can solve the model in this paper well, and the convergence is good.

(2) The total distribution cost, vehicle use and labor cost, refrigeration cost, and vehicle use number of $C$-type dataset are the highest among all types, but the fuel consumption cost is the lowest compared with other types. The main reason is that the $C$-type distribution customer points are mainly concentrated in several areas and the driving distance is short, so the fuel consumption cost is relatively low. However, the customer time window is relatively narrow. As the customer point service time of the $C$-type dataset in Solomon's database is $90 \mathrm{~min}$, which is different from the 10 min service time of $R$ and RC customers, the vehicle can only serve fewer customers, and the total delivery travel time is longer.

(3) The total distribution cost, vehicle rental and labor cost, and number of vehicles of $R$-type and RC-type categories are less than those of $C$-type categories, 
TABLE 3: The vehicle path planning of RC204 dataset.

\begin{tabular}{|c|c|c|}
\hline SN & VR & VAT \\
\hline 1 & $0-1-2-6-7-3-5-45-46-16-15-10-11-0$ & $\begin{array}{l}0-37.98-57.92-73.85-90.25-125.02-139.01-155.01-171.12-228.94-240.79-260.52- \\
272.72-305.02\end{array}$ \\
\hline 2 & $\begin{array}{l}0-8-73-47-17-9-12-14-59-97-24-22-13-18- \\
19-20-49-21-48-25-77-58-0\end{array}$ & $\begin{array}{c}326.83-366.21-401.42-426.22-438.68-462.00-481.22-496.22-529.97-546.69-590.98- \\
603.25-654.63-755.24-775.63-792.74-807.75-824.82-836.02-853.04-874.97-896.24- \\
943.77\end{array}$ \\
\hline 3 & $\begin{array}{l}0-23-51-85-26-28-30-32-27-29-31-33-34-35- \\
36-37-0\end{array}$ & $\begin{array}{c}131.4-200.99-236.53-253.28-295.83-310.03-324.03-356.38-375.48-391.27-401.27- \\
423.79-441.97-506.03-518.06-531.03-572.63\end{array}$ \\
\hline 4 & $0-38-39-40-41-42-43-0$ & $239.27-277.87-288.92-304.42-3$ \\
\hline 5 & $\begin{array}{l}0-44-50-63-76-89-64-52-53-54-81-72-71-55- \\
56-57-74-86-75-61-68-0\end{array}$ & $\begin{array}{l}182.77-218.1-277.03-304.49-323.01-343.21-387.07-417.17-447.75-492.17-508.43- \\
534.24-554.43-601.74-641.76-678.46-712.82-731.07-788.76-872.03-889.78-897.94\end{array}$ \\
\hline 6 & $0-62-95-65-66-80-91-67-0$ & $107.9-168-194.03-225.78-242.18$ \\
\hline 7 & $0-69-70-88-98-82-83-90-99-87-79-84-0$ & $\begin{array}{c}137.83-158-195.52-223.14-239.42-258.12-282.89-308.83-334.34-364.23-418.42-492.82- \\
517.94\end{array}$ \\
\hline 0 & & $0-14.65-35.72-53.47-69.79-98.93$ \\
\hline 0 & $0-4-60-78-100-0$ & 608.03-643.98-680.41-704.24-780.97-799.62 \\
\hline
\end{tabular}

TABLE 4: Experimental data results of different distribution examples.

\begin{tabular}{lcccccc}
\hline EX & C103 & C104 & R203 & R204 & RC204 & RC208 \\
\hline TC/yuan & 17180.60 & 15914.90 & 13386.20 & 11642.70 & 10051.90 & 11504.80 \\
TMC/min & 6889.20 & 6553.40 & 4460.00 & 3946.90 & 2843.00 & 3493.00 \\
CC/yuan & 5698.90 & 5007.50 & 3866.90 & 3057.00 & 2655.80 \\
FC/yuan & 3822.90 & 3785.94 & 4727.10 & 4050.50 & 3903.40 & 3450.90 \\
CF/yuan & 48.70 & 45.40 & 61.10 & 52.50 & 50.00 & 53.80 \\
PC/yuan & 506.70 & 506.70 & 506.70 & 432.20 & 394.60 & 228.50 \\
UC/yuan & 210.49 & 248.88 & 66.74 & 99.80 & 101.30 & 107.85 \\
VN & 25 & 23 & 15 & 60.16 & 54.70 \\
((TMC+CC)/TC)/\% & 73.27 & 72.64 & 62.21 & 34.79 & 38.83 \\
(FC/TC)/\% & 22.25 & 23.79 & 35.31 & 0.45 & 0.50 & 36.36 \\
(CF/TC)/\% & 0.28 & 0.29 & 0.46 & 0.46 \\
\hline
\end{tabular}

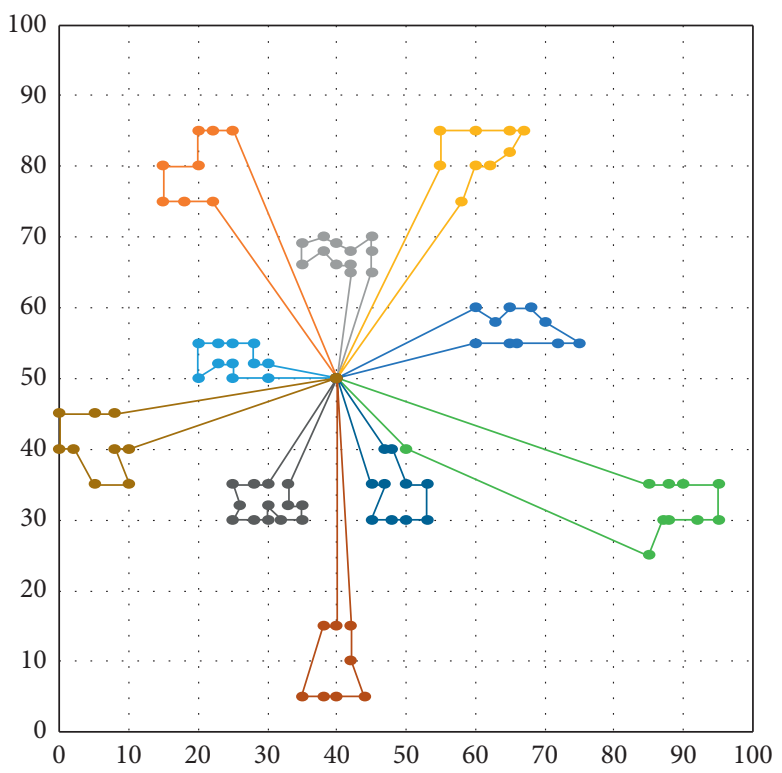

(a)

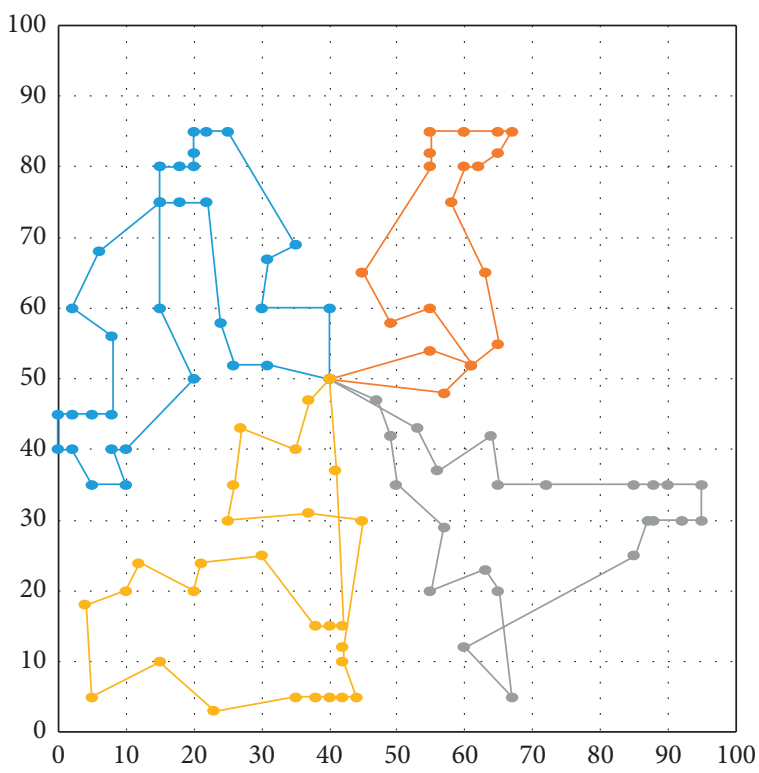

(b)

Figure 2: Example of vehicle path planning. (a) C104. (b) RC208. 
mainly because the service time of these two types is only $10 \mathrm{~min}$, customers are randomly distributed, and the time window requirements of customers are relatively loose, so the vehicles can deliver to multiple customers, and the total driving time is shorter.

(4) In the total cost composition of distribution, all types of TMC and CC account for a relatively high proportion, accounting for more than half of the total cost, while $C$ accounts for more than $70 \%$. This shows that the cost of logistics distribution mainly comes from vehicle use cost, manpower cost, and refrigeration cost. The main influencing factor is the travel time, so to reduce the logistics cost, the most important thing is to reduce the total transportation time.

(5) The proportion of fuel consumption cost in logistics is low, the highest is only about $38 \%$ of the total cost of RC-type dataset, and the lowest is only about $22 \%$ of $C$-type dataset. The carbon emission ratio is very low, accounting for only $0.2 \%$ to $0.4 \%$ of the total cost. It shows that from the economic point of view of logistics enterprises, the carbon emission cost generated by the current carbon tax can hardly effectively promote the energy conservation and emission reduction of enterprises.

4.4. Simulation under Different Traffic Factors. On the premise that other conditions of the algorithm remain unchanged, the traffic factors $r_{i j}$ are set as $1.5,3.5$, and 8.5 to simulate the situation of temperature measuring jam, traffic jam, and road closure. Then, the path planning was performed on RC204 dataset. Each case was solved for 10 times, and the average value was taken as the final result. The optimized results obtained are shown in Table 5, where CP represents the carbon emissions and TT represents the traveling time.

By observing the recognition results in Table 5, the following can be seen:

(1) With the increase of traffic factors, the total cost of distribution, vehicle use cost, labor cost, and refrigeration cost will increase to varying degrees, indicating that these costs are positively correlated with congestion.

(2) With the increase of traffic factor, fuel consumption and carbon emissions increase, indicating that congestion will affect the greenness of cold chain logistics distribution path. The driving time also increases, indicating that traffic congestion will affect the speed and thus affect vehicle path planning.

(3) Although traffic factors change significantly, TC, TMC, CC, FC, CP, and VN have little change because the model constructed in this paper can make most vehicles effectively avoid the traffic jam period, which demonstrates the effectiveness of the model and solving algorithm in this paper.
TABLE 5: Simulation results under different traffic factors.

\begin{tabular}{lccc}
\hline Traffic factors $r_{i j}$ & 1.5 & 3.5 & 8.5 \\
\hline TC/yuan & 9871.5 & 1051.7 & 10383.1 \\
TMC/min & 2798.6 & 2842.8 & 2960.3 \\
CC/yuan & 2647.1 & 2655.5 & 2689.2 \\
CF/yuan & 3888.4 & 3903 & 4005.1 \\
PC/yuan & 905.6 & 947.7 & 990.1 \\
UC/yuan & 98 & 101.3 & 106.7 \\
VN & 9 & 9 & 10 \\
TT/min & 4759 & 4836 & 4902 \\
\hline
\end{tabular}

\section{Conclusion}

Under the background of normal epidemic situation, the contactless distribution of fresh goods has important research significance. Reasonable planning of fresh goods logistics distribution path can not only reduce the distribution cost in logistics, but also increase customer satisfaction. This paper studies the possible traffic factors in the epidemic environment and comprehensively considers the vehicle management cost, fuel consumption and carbon emission cost, refrigeration cost, time window penalty cost, and user satisfaction cost. An optimization model for this problem is built, and an improved ant colony algorithm model based on path partitioning strategy is designed. The heuristic factor and pheromone updating strategy of basic ant colony algorithm are improved. The simulation results show that the model can deal with various traffic factors effectively. The proposed algorithm can achieve better optimization results in different distributed data cases.

\section{Data Availability}

The labeled dataset used to support the findings of this study are available from the corresponding author upon request.

\section{Conflicts of Interest}

The author declare that they have no conflicts of interest regarding the publication of this paper.

\section{Acknowledgments}

This study was supported by Henan Province Science and Technology Research Project (Project no. 172102210450) and Xinyang Agriculture and Forestry College Young Teacher Fund Project (2018LG015).

\section{References}

[1] C. Luo, L. Wu, and N. Liu, "Study based on contactless distribution patterns under the outbreak," IOP Conference Series: Earth and Environmental Science. IOP Publishing, vol. 526, no. 1, Article ID 12204, 2020.

[2] G. B. Dantzig and J. H. Ramser, "The truck dispatching problem,” Management Science, vol. 6, no. 1, pp. 80-91, 1959.

[3] M. Desrochers and T. W. Verhoog, "A new heuristic for the fleet size and mix vehicle routing problem," Computers \& Operations Research, vol. 18, no. 3, pp. 263-274, 1991. 
[4] M. M. Solomon and J. Desrosiers, "Survey paper-time window constrained routing and scheduling problems," Transportation Science, vol. 22, no. 1, pp. 1-13, 1988.

[5] O. Jabali, R. Leus, T. Van Woensel, and T. de Kok, "Selfimposed time windows in vehicle routing problems," $O R$ Spectrum, vol. 37, no. 2, pp. 331-352, 2015.

[6] R.-M. Chen, Y.-M. Shen, and W.-Z. Hong, "Neural-like encoding particle swarm optimization for periodic vehicle routing problems," Expert Systems with Applications, vol. 138, Article ID 112833, 2019.

[7] R. Kramer, A. Subramanian, T. Vidal, and L. d. A. F. Cabral, "A matheuristic approach for the pollution-routing problem," European Journal of Operational Research, vol. 243, no. 2, pp. 523-539, 2015.

[8] Y. Xiao and A. Konak, "The heterogeneous green vehicle routing and scheduling problem with time-varying traffic congestion," Transportation Research Part E: Logistics and Transportation Review, vol. 88, pp. 146-166, 2016.

[9] Q. K. Cao, K. W. Yang, and X. Y. Ren, "Vehicle routing optimization with multiple fuzzy time windows based on improved wolf pack algorithm," Advances in Production Engineering \& Management, vol. 12, no. 4, pp. 401-411, 2017.

[10] R. Yan, X. N. Zhu, and Q. Zhang, "Research of the model and algorithm for two-dimensional multi-depots capacitated vehicle routing problem with time window constrain," Chinese Journal of Management Science, vol. 25, no. 7, pp. 67-77, 2017.

[11] S. Huber and M. J. Geiger, "Order matters-a variable neighborhood search for the swap-body vehicle routing problem," European Journal of Operational Research, vol. 263, no. 2, pp. 419-445, 2017.

[12] J. D. Camm, M. J. Magazine, S. Kuppusamy, and K. Martin, "The demand weighted vehicle routing problem," European Journal of Operational Research, vol. 262, no. 1, pp. 151-162, 2017.

[13] C. D. Tarantilis and C. T. Kiranoudis, "A meta-heuristic algorithm for the efficient distribution of Perishable foods," Journal of Food Engineering, vol. 50, no. 1, pp. 1-9, 2001.

[14] P. Amorim, S. N. Parragh, F. Sperandio, and B. Almada-Lobo, "A rich vehicle routing problem dealing with perishable food: a case study," TOP, vol. 22, no. 2, pp. 489-508, 2014.

[15] J. Yang and H. Sun, "Battery swap station location-routing problem with capacitated electric vehicles," Computers \& Operations Research, vol. 55, pp. 217-232, 2015.

[16] Y. M. Zhang, Y. M. Li, and H. O. Liu, "Research on VRP optimization of multi-type vehicle cold-chain logistics with satisfaction constraint," Statistics and Decision, vol. 35, no. 4, pp. 176-181, 2019.

[17] X. G. Ma, T. J. Liu, P. Z. Yang et al., "Vehicle routing optimization model of cold chain logistics based on stochastic demand," Journal of System Simulation, vol. 8, pp. 1824-1832, 2016.

[18] H. Lan, Q. F. He, and Z. Bian, "Distribution routing optimization of cold chain logistics with consideration of road traffic conditions," Journal of Dalian Maritime University, vol. 41, no. 11, pp. 67-74, 2015.

[19] N. Li and S. B. Wang, "Production and distribution optimization model for perishable products in uncertain demand," Application Reseach of Computers, vol. 28, no. 3, pp. 927-929, 2011.

[20] J. Dai, L. Xie, and Q. Wang, "The impact of the third party logistics integration on logistics service quality, partnership and corporate operational performance," Management Review, vol. 27, no. 5, pp. 188-197, 2015.
[21] H. B. Duan, Ant Colony Algorithms: Theory and Applications, pp. 33-38, Science Press, Beijing, China, 2005.

[22] A. J. Hickman, "Methodology for calculating transport emissions and energy consumption," Report, Transport Research Laboratory, Crowthorne, UK, 1999. 